\title{
Magnetic and Superfluid Transitions in the d=1 Spin-1 Boson Hubbard Model
}

\author{
G.G. Batrouni ${ }^{1}$, V.G. Rousseau ${ }^{2}$, and R.T. Scalettar ${ }^{3}$ \\ ${ }^{1}$ INLN, Université de Nice-Sophia Antipolis, CNRS; 1361 route des Lucioles, 06560 Valbonne, France \\ ${ }^{2}$ Instituut-Lorentz, LION, Universiteit Leiden, Postbus 9504, 2300 RA Leiden, The Netherlands and \\ ${ }^{3}$ Physics Department, University of California, Davis, CA 95616
}

\begin{abstract}
The interplay between magnetism and metal-insulator transitions is fundamental to the rich physics of the single band fermion Hubbard model. Recent progress in experiments on trapped ultra-cold atoms have made possible the exploration of similar effects in the boson Hubbard model (BHM). We report on Quantum Monte Carlo (QMC) simulations of the spin-1 BHM in the ground state. For antiferromagnetic interactions, $\left(U_{2}>0\right)$, which favor singlet formation within the Mott insulator lobes, we present exact numerical evidence that the superfluid-insulator phase transition is first (second) order depending on whether the Mott lobe is even (odd). Inside even Mott lobes, we examine the possibility of nematic-to-singlet first order transitions. In the ferromagnetic case $\left(U_{2}<0\right)$, the transitions are all continuous. We map the phase diagram for $U_{2}<0$ and demonstrate the existence of the ferromagnetic superfluid. We also compare the QMC phase diagram with a third order perturbation calculation.
\end{abstract}

PACS numbers: 05.30.Jp, 03.75.Hh, 67.40.Kh, 75.10.Jm 03.75.Mn

The single band fermion Hubbard model (FHM) offers one of the most fundamental descriptions of the physics of strongly correlated electrons in the solid state. The spinful nature of the fermions is central to the wide range of phenomena it displays such as interplay between its magnetic and transport properties. ${ }^{1}$ Such complex interplay is absent in the superfluid to Mott insulator transition 2.3,4,5 in the spin-0 Boson Hubbard model (BHM). However, purely optical traps ${ }^{6}$ can now confine alkali atoms ${ }^{23} \mathrm{Na},{ }^{39} \mathrm{~K}$, and ${ }^{87} \mathrm{Rb}$, which have hyperfine spin $F=1$, without freezing $F_{z}$. As in the fermion case, the nature of the superfluid-Mott insulator (SF-MI) transition is modified by the spin fluctuations which are now allowed. Initial theoretical work employed continuum, effective low-energy Hamiltonians and determined the magnetic properties and excitations of the superfluid phases $?^{7}$

To capture the SF-MI transition it is necessary to consider the spin-1 Bosonic Hubbard Hamiltonian,

$$
\begin{aligned}
H= & -t \sum_{\langle i j\rangle, \sigma}\left(a_{i \sigma}^{\dagger} a_{j \sigma}+h . c\right)+\frac{U_{0}}{2} \sum_{i} \hat{n}_{i}\left(\hat{n}_{i}-1\right) \\
& +\frac{U_{2}}{2} \sum_{i}\left(\vec{F}_{i}^{2}-2 \hat{n}_{i}\right)
\end{aligned}
$$

The boson creation (destruction) operators $a_{i \sigma}^{\dagger}\left(a_{i \sigma}\right)$ have site $i$ and spin $\sigma$ indices. $\sigma=1,0,-1$. The first term describes nearest-neighbor, $\langle i j\rangle$, jumps. The hybridization $t=1$ sets the energy scale, and we study the one dimensional case. The number operator $\hat{n}_{i} \equiv \sum_{\sigma} \hat{n}_{i \sigma}=$ $\sum_{\sigma} a_{i \sigma}^{\dagger} a_{i \sigma}$ counts the total boson density on site $i$. The on-site repulsion $U_{0}$ favors states with uniform occupation and competition between $U_{0}$ and $t$ drives the MI-SF transition. The spin operator $\vec{F}_{i}=\sum_{\sigma, \sigma^{\prime}} a_{i \sigma}^{\dagger} \vec{F}_{\sigma \sigma^{\prime}} a_{i \sigma^{\prime}}$, with $\vec{F}_{\sigma \sigma^{\prime}}$ the standard spin-1 matrices, contains further density-density interactions and also interconversion terms between the spin species. We treat the sys- tem in the canonical ensemble where the total particle number is fixed and the chemical potential is calculated, $\mu(N)=E(N+1)-E(N)$ where $E(N)$ is the ground state energy with $N$ particles. This holds only when each energy used is that of a single thermodynamic phase and not that of a mixture of coexisting phases as happens with first order transitions. It is therefore incorrect to determine first order phase boundaries with the naive use of this method. In the present case there is the added subtlety that the three species are interconvertible. These issues will be addressed in more detail below.

Several important aspects of the spin- 1 BHM are revealed by analysing the independent site limit, $t / U_{0}=0$. The Mott- 1 state with $n_{i}=1$ on each site has $\mathcal{E}_{\mathrm{M}}(1)=0$. In the Mott-2 state with $n_{i}=2$, the energy is $\mathcal{E}_{\mathrm{M}}(2)=$ $U_{0}-2 U_{2}$, if the bosons form a singlet, $F=0$, and is $\mathcal{E}_{\mathrm{M} 2}=U_{0}+U_{2}$ if $F=2$. Thus $U_{2}>0$ favors singlet phases while $U_{2}<0$ favors (on-site) ferromagnetism. This applies to all higher lobes as well. In the canonical ensemble, the chemical potential at which the system goes from the $n$th to the $(n+1)$ th Mott lobe is $\mu(n \rightarrow n+1)=\mathcal{E}_{n+1}-\mathcal{E}_{n}$.

First consider $U_{2}>0$. The energy of Mott lobes at odd filling, $n_{\mathrm{o}}$, is $\mathcal{E}_{M}\left(n_{\mathrm{o}}\right)=U_{0} n_{\mathrm{o}}\left(n_{\mathrm{o}}-1\right) / 2+U_{2}\left(1-n_{\mathrm{o}}\right)$ while at even filling, $n_{\mathrm{e}}, \mathcal{E}_{M}\left(n_{\mathrm{e}}\right)=U_{0} n_{\mathrm{e}}\left(n_{\mathrm{e}}-1\right) / 2-n_{\mathrm{e}} U_{2}$. Therefore, the boundaries of the lobes, going from lower to higher filling, are $\mu\left(n_{\mathrm{e}} \rightarrow\left(n_{\mathrm{e}}+1\right)\right)=n_{\mathrm{e}} U_{0}$ and $\mu\left(n_{\mathrm{o}} \rightarrow\right.$ $\left.\left(n_{\mathrm{o}}+1\right)\right)=n_{\mathrm{o}} U_{0}-2 U_{2}$. This demarks the positions of the 'bases' of the Mott lobes in the $\left(t / U_{0}, \mu / U_{0}\right)$ ground state phase diagram. For $U_{2}>0$ the even Mott lobes grow at the expense of the odd ones, which disappear entirely for $U_{0}=2 U_{2}$.

For $U_{2}<0$, the ground state is ferromagnetic (maximal $F: F^{2}=n(n+1)$ ) which gives for all Mott lobes $\mathcal{E}_{\mathrm{M}}(n)=n(n-1)\left(U_{0}+U_{2}\right) / 2$. Consequently, the boundary of the $n$th and $(n+1)$ th Mott lobes $\mu(n \rightarrow n+1)=$ $n\left(U_{0}+U_{2}\right)$. The bases of both the odd and even Mott lobes shrink with increasing $\left|U_{2}\right|$, in contrast to the 
$U_{2}>0$ case where the even Mott lobes expand.

Mean-field treatments of the lattice model capture the SF-MI transition as the hopping $t$ is turned on, and have been performed both at zero and finite temperature. $., 9,10$ Even when $U_{2}=0$, the spin degeneracy alters the nature of the transition. For $U_{2}>0$, the order of the phase transition depends on whether the Mott lobe is even or odd. These mean field calculations assume a non-zero order parameter $\left\langle a_{i \sigma}\right\rangle$, which cannot be appropriate in $\mathrm{d}=1$ or in $\mathrm{d}=2$ at finite $T$. Therefore it is important to verify these predictions for the qualitative aspects of the phase diagram, especially in low dimension. A quantitative determination of the phase boundaries requires numerical treatments. Indeed, $\mathrm{DMRG}^{11}$ and Quantum Monte Carlo (QMC) $\frac{12}{\underline{ }}$ results for $U_{2}>0$ and $\mathrm{d}=1$ reported the critical coupling strength and showed the odd Mott lobes are characterized by a dimerized phase which breaks translation symmetry.

For $U_{2}<0$, the nature of the SF-MI transition does not depend on the order of the Mott lobe while for $U_{2}>$ 0 it is predicted to be continuous (discontinuous) into odd (even) lobes. Consequently, it suffices to study the first two Mott lobes both for $U_{2}>0$ and $U_{2}<0$ to demonstrate the behavior for all lobes. Furthermore, in what follows we will focus on the case $\left|U_{2} / U_{0}\right|=0.1$ in order to compare our results for $U_{2}>0$ with Rizzi et al11

Here, we will use an exact QMC approach, the SGF algorithm with directed update, to study the spin-1 BHM in $\mathrm{d}=1$ for both positive and negative $U_{2} \underline{\underline{13}}$

For $U_{2}>0\left(\right.$ e.g. $\left.{ }^{23} \mathrm{Na}\right)$, which favors low total spin states, Fig. 1 1 shows the total number density $\rho=N / L$ against the chemical potential, $\mu$, for $U_{0}=10 t$ and $U_{2}=t$. It displays clearly the first two incompressible MI phases. In agreement with the $t / U_{0}=0$ analysis, $U_{2}$ causes an expansion of the second Mott lobe, $\rho=2$, at the expense of the first, $\rho=1$. Our Mott gaps agree with DMRG results 11 to within symbol size. However, the $\rho$ versus $\mu$ curve in Fig. 1 does not betray any evidence of the different natures of the phase transitions into the first and second Mott lobes. In particular, for a spin-0 BHM, first order transitions are clearly exposed by the appearence of negative compressibility $\stackrel{14}{\Perp} \kappa=\partial \rho / \partial \mu$, which is not present here. The transition into the second Mott lobe is expected to be first order and driven by the formation of bound pairs of bosons in singlet states. Therefore, in the canonical ensemble, we expect that near this transition there will be phase coexistence between singlets arranged in a Mott region and superfluid.

The nature of the transitions is revealed by the evolution of the spin populations in the system as $\rho$ increases. Since the singlet wavefunction is $|0,0\rangle=$ $\sqrt{2 / 3}|1,1\rangle|1,-1\rangle-\sqrt{1 / 3}|1,0\rangle|1,0\rangle$, this state has $\rho_{+}=$ $\rho_{0}=\rho_{-}$. We plot in the inset of Fig. 1 the population fractions, $N_{0} / N$ and $N_{-} / N=N_{+} / N$ versus the total density. We see that as $\rho$ increases, $N_{+} / N$ and $N_{0} / N$ oscillate: When $N$ is even, singlet bound states of two particles try to form drawing the values of $N_{+} / N$ and $N_{0} / N$ closer together. However, singlets form fully, mak-

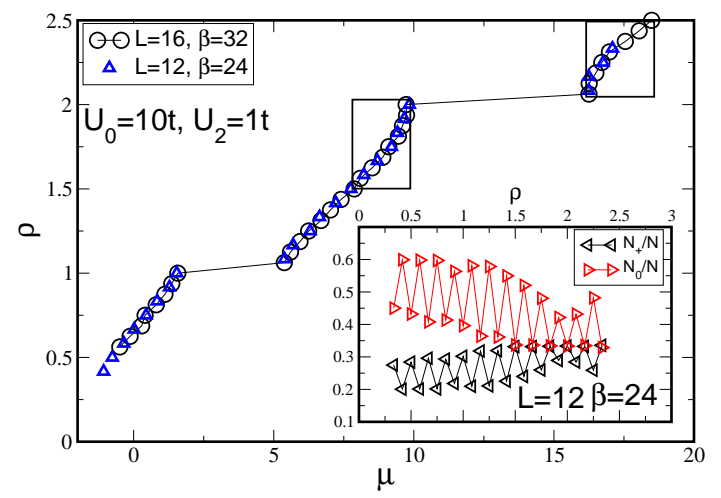

FIG. 1: (Color online) $\rho(\mu)$ exhibits Mott plateaux: gapped, insulating phases at commensurate fillings. Inset: $N_{+} / N$ and $N_{0} / N$ vs $\rho=N / L$. Singlets form where $N_{+} / N$ and $N_{0} / N$ are equal (see text).

ing $N_{+}=N_{0}$, only close to the second Mott lobe, $\rho=2$, where we clearly see $N_{+} / N=N_{0} / N$ for a range of even values of $N$. On the other hand, when $N$ is odd, singlets cannot form and the spin populations are much farther from that given by the singlet wavefunction. In the thermodynamic limit and fixed $N$, one expects true phase separation into $\rho=2$ singlet MI regions and $\rho<2$ SF regions. For a finite system, phase separation commences for (even) fillings where we first have $N_{+} / N=N_{0} / N$, i.e. $1.5 \leq \rho<2$ and similar behavior for $\rho>2$. Another interesting feature in the inset of Fig. 1 is that the difference between $N_{+} / N$ and $N_{0} / N$, for even $N$, decreases linearly as the density approaches the transition at $\rho=1.5$. No such behavior is seen as the first Mott lobe is entered from below or above $\rho=1$ : the transition is coninuous as predicted for odd lobes.

The boxes in Fig. 1 1 show the values of $\rho$ corresponding to phase coexistence rather than to one stable thermodynamic phase. It is, therefore, clear that the canonical calculation of the phase boundaries, i.e. simply adding a particle to, or removing it from, the MI, is not applicable in the presence of a first order transition.

Figure 1 reveals the SF-MI transition at fixed, sufficiently large $U_{0}$ when $\rho$ is varied. In Fig. 2 we show the transition when the density is fixed, $\rho=2$, and $U_{0}$ is the control parameter with $U_{2} / U_{0}$ fixed at 0.1 (main figure) and 0.01 (inset). Singlet formation is clearly shown by $\left\langle F^{2}\right\rangle \rightarrow 0$, as $t / U_{0}$ decreases and the second $\mathrm{MI}$ is entered,$\frac{15}{n}$ Indeed, the origin of the first order transition into even Mott lobes, as the filling is tuned, is linked to the additional stabilization of the Mott lobe associated with this singlet energy $\underline{10}$ The superfluid density, $\rho_{s}=L\left\langle W^{2}\right\rangle / 2 t \beta$, where $W$ is the winding number, is a topological quantity and truly characterizes the SF-MI phase transition which is continuous in this case. As $L$ is increased from 10 to 16 and 20, the vanishing of $\rho_{s}$ gets sharper. We find that the critical value of $t / U_{0}$ for the $\rho=2$ lobe is somewhat less than that reported in DMRG 11 indicated by the dashed line. We believe this 


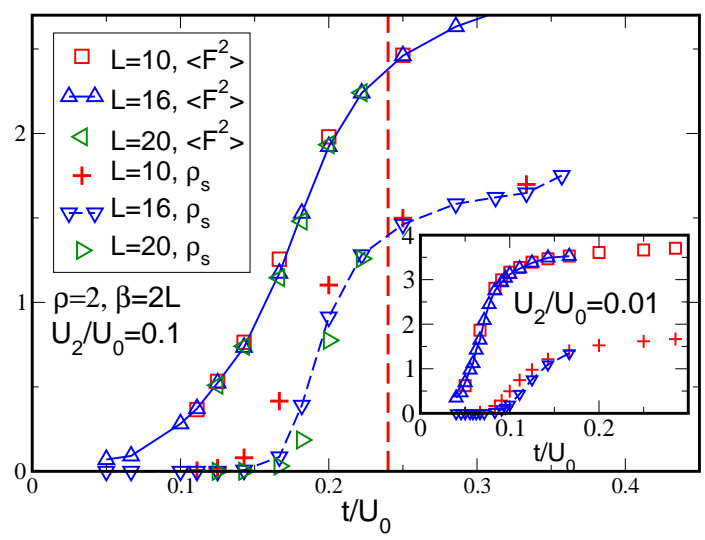

FIG. 2: (Color online) The square of the local moment, $\left\langle F^{2}\right\rangle$, and the superfluid density, $\rho_{s}$, vs $t / U_{0}$ for $\rho=2$. In the Mott lobe, $\left\langle F^{2}\right\rangle \rightarrow 0$ signaling singlet formation and $\rho_{s} \rightarrow 0$ indicating an insulator. The dashed line indicates the critical $t / U_{0}$ from Rizzi et $a^{111}$. Inset: Same but with $U_{2} / U_{0}=0.01$.

is because with DMRG the phase boundaries were obtained using finite differences of the energy with small doping above and below commensurate filling. As discussed above, this is not appropriate for a first order transition.

For $2 d U_{2} / U_{0}<0.1$, and $d=2,3$, mean field ${ }^{18,19}$ predicts that, when $t / U_{0}^{c 1} \sim \sqrt{U_{2} / 4 d U_{0}}$ is in the MI, then Mott lobes of even order are comprised of two phases: (a) the singlet phase for $t / U_{0} \leq t / U_{0}^{c 1}$ and (b) a nematic phase for $t / U_{0}^{c 1} \leq t / U_{0} \leq t / U_{0}^{c}$, where $t / U_{0}^{c}$ is the tip of the Mott lobe. Inside the lobe, the nematic-to-singlet transition is predicted to be first order which raises the question: are the singlet-to-SF and the nematic-to-SF transitions of the same order? Figure 2 shows that the SF-MI transition, $\rho_{s} \rightarrow 0$, occurs at larger $t / U_{0}$ than singlet formation, $\left\langle F^{2}\right\rangle \rightarrow 0$, both for $U_{2} / U_{0}=0.1$ and 0.01. The passage of $\left\langle F^{2}\right\rangle$ to zero gets sharper for smaller $U_{2} / U_{0}$ but remains continuous, not exhibiting any signs of a first order transition. We have verified this for $U_{2} / U_{0}=0.1,0.05,0.01,0.005$. Furthermore, the insensitivity of $\left\langle F^{2}\right\rangle$ to finite size effects indicates that it does not undergo a continuous phase transition. We have also verified that for the second Mott lobe, the SF-MI transition is first order regardless whether $t / U_{0}$ is less or greater than $t / U_{0}^{c 1}$. We conclude that while $\rho_{s} \rightarrow 0$ is a continuous critical transition, $\left\langle F^{2}\right\rangle \rightarrow 0$ is a crossover not a phase transition. This, of course, does not preclude the possibility of a first order transition for $d=2,3$.

Whereas ${ }^{23} \mathrm{Na}$ has positive $U_{2},{ }^{87} \mathrm{Rb}$ has $U_{2}<0$, leading to different behavior. We begin with $\rho$ versus $\mu$ in Fig. 3 Unlike the $U_{2}>0$ case, the SFMI transitions are continuous for both even and odd Mott lobes: the inset shows that the spin populations do not oscillate as for $U_{2}>0$. The population ratio, $\rho_{0}=2 \rho_{+}$can be understood as follows. As shown above for $t / U_{0} \rightarrow 0$, maximum spin states are favored when $U_{2}<0$. So, when a site is doubly occupied, the

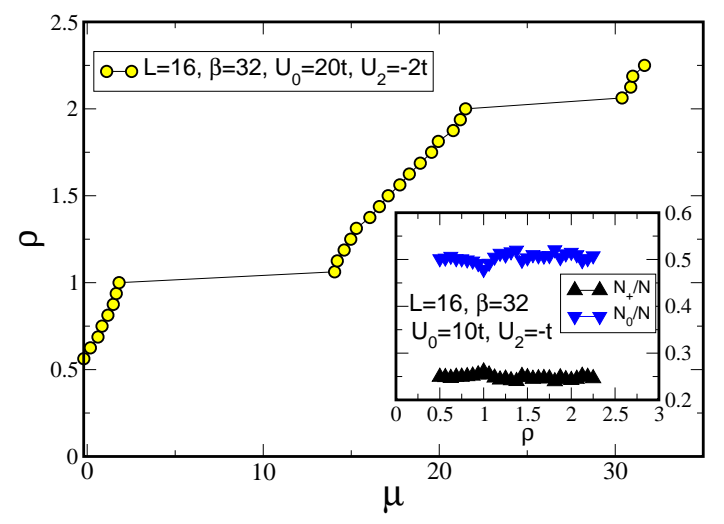

FIG. 3: (Color online) The density, $\rho$ versus chemical potential, $\mu$, exhibits the usual Mott plateaux at commensurate filling for $U_{2}<0$. Inset: The spin population fractions showing $\rho_{0}=2 \rho_{+}$. They do not exhibit oscillations like in Fig. 1.

spin-2 state is favored. But, since our study is in the $S_{z}^{\text {total }}=N_{+}-N_{-}=0$ sector, the wavefunction of the spin-2 state is $|2,0\rangle=1 / \sqrt{3}|1,1\rangle|1,-1\rangle+\sqrt{2 / 3}|1,0\rangle|1,0\rangle$ and thus $\rho_{0}=2 \rho_{+}=2 \rho_{-}$.

As discussed above, $U_{2}<0$ favors 'local ferromagnetism', namely high spin states on each of the individual lattice sites. As with the FHM, the kinetic energy gives rise to second order splitting which lifts the degeneracy between commensurate filling strong coupling states with different intersite spin arrangements. We can therefore ask whether the local moments order from site to site: Do the Mott and superfluid phases exhibit global ferromagnetism $\frac{10}{2}$ ? To this end, we measure the magnetic structure factor,

$$
S_{\sigma \sigma}(q)=\sum_{l} e^{i q l}\left\langle F_{\sigma, j+l} F_{\sigma, j}\right\rangle
$$

where $\sigma=x$ or $z$. Figure 4 shows $S_{\mathrm{xx}}(q)$ in the superfluid phase at half-filling $\underline{\underline{16}}$ The peak at $q=0$ grows linearly with lattice size, indicating the superfluid phase does indeed possess long range ferromagnetic order. We find that the MI phase is also ferromagnetic.

To determine the phase diagram, we scan the density as in Fig. 3 for many values of $U_{0}$ with $U_{2} / U_{0}$ constant $(-0.1$ in our case). The resulting phase diagram is shown in Fig. 5. Comparison of data for two lattice sizes demonstrates that finite size effects are small.

Early in the evaluation of the phase boundaries of the spin-0 BHM it was observed that a perturbation calculation ${ }^{4}$ agreed remarkably well with QMC results .3 We now generalize the spin-0 perturbation theory to spin1 and show a similar level of agreement with the QMC results. If we assume the system always to be perfectly magnetized, then $n$ bosons on a site will yield the largest possible spin, $F^{2}=n(n+1)$. Consequently, the interaction term in the Hamiltonian, Eq.(11), reduces to $\left(U_{0}-U_{2}\right) \sum_{i} \hat{n}_{i}\left(\hat{n}_{i}-1\right) / 2$, giving a Hamiltonian identical to the spin-0 BHM but with the interaction shifted to $\left(U_{0}-U_{2}\right) / 2$. One can then repeat the perturbation 


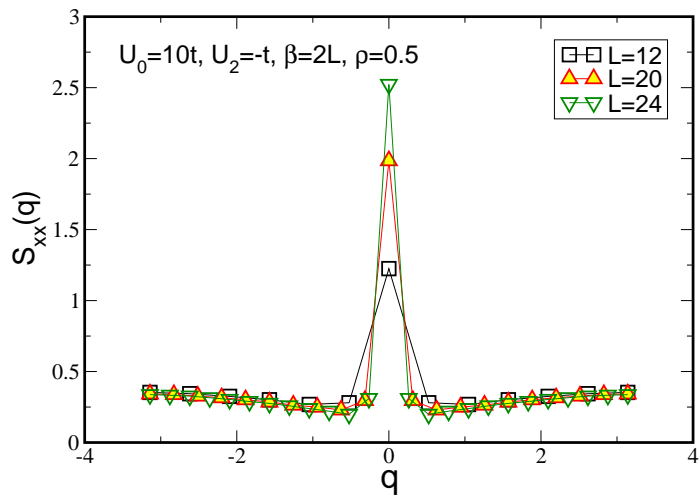

FIG. 4: (Color online) The magnetic structure factor $S_{\mathrm{xx}}(q)$ for $U_{2}<0$ and $\rho=0.5$ exhibits a sharp $q=0$ peak indicating ferromagnetic order.

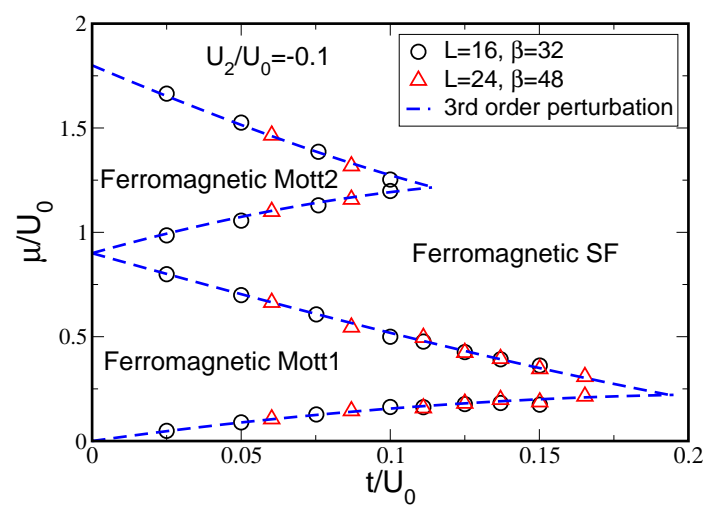

FIG. 5: (Color online) Phase diagram of the spin-1 BHM for $U_{2} / U_{0}=-0.1$.

expansion to third order in $t /\left(U_{0}-U_{2}\right)$ to determine the phase diagram $\stackrel{4}{\underline{4}}$ The result is shown as the dashed line in Fig. 5 and is seen to be in excellent agreement with QMC. The agreement further suggests that the finite lattice effects in the phase diagram are small. Such a perturbation calculation is not possible for the $U_{0}>0$ case since $F^{2}$ depends on the phase, SF vs MI, and on the order of the MI lobe.

The (dipolar) interactions between spinful bosonic atoms confined to a single trap have been shown to give rise to fascinating "spin textures" 17 An additional optical lattice causes a further enhancement of interactions, and opens the prospect for the observation of the rich behavior associated with Mott and magnetic transitions, and comparisons with analogous properties of strongly correlated solids $\stackrel{18,19}{ }$ Here, we have quantified these phenomena in the one-dimensional spin-1 BHM with exact QMC methods. We have shown that, for $U_{2}>0$, the MI phase is characterized by singlet formation clearly seen for even Mott lobes where $\left\langle F^{2}\right\rangle \rightarrow 0$ as $U_{0}$ increases. We also showed that the transition into odd lobes is continuous while that into even lobes is discontinuous (first order). We emphasized that the naive canonical determination of the phase boundaries is not appropriate for a first order transition. For $U_{2}<0$, we showed that all MISF transitions are continuous and that both the SF and MI phases are ferromagnetic. The phase diagram in the $\left(\mu / U_{0}, t / U_{0}\right)$ plane obtained by QMC can be described very accurately using third order perturbation theory.

Acknowledgements: G.G.B. is supported by the CNRS (France) PICS 3659, V.G.R. by the research program of the 'Stichting voor Fundamenteel Onderzoek der Materie (FOM)' and R.T.S. by ARO Award W911NF0710576 with funds from the DARPA OLE Program. We would like to thank T.B. Bopper for useful input.
1 "Lecture Notes on Electron Correlation and Magnetism," Patrik Fazekas, World Scientific (1999).

2 M.P.A. Fisher et al., Phys. Rev. B40, 546 (1989).

${ }^{3}$ G.G. Batrouni, R.T. Scalettar, and G.T. Zimanyi, Phys. Rev. Lett. 65, 1765 (1990).

4 J.K. Freericks and H. Monien, Phys. Rev. B53, 2691 (1996).

5 B. Capogrosso-Sansone et al, Phys. Rev. A77 015602 (2008).

6 D.M. Stamper-Kurn et al, Phys. Rev. Lett. 80, 2027 (1998).

7 T.L. Ho, Phys. Rev. Lett. 81, 742 (1998); T. Ohmi and K. Machida, J. Phys. Soc. Japan 67, 1822 (1998); S. Mukerjee, C. Zu, and J.E. Moore, Phys. Rev. Lett. 97, 120406 (2006).

8 K.V. Krutitsky and R. Graham, Phys. Rev. A70, 063610 (2004); S. Ashhab, J. Low Temp. Phys. 140, 51 (2005).

9 T. Kimura, S. Tsuchiya, and S. Kurihara Phys. Rev. Lett. 94, 110403 (2005).

10 V. Pai, K. Sheshadri and R. Pandit, Phys. Rev. B77, 014503 (2008).

11 M. Rizzi et al, Phys. Rev. Lett. 95, 240404 (2005); S.
Bergkvist, I. McCulloch and A. Rosengren, Phys. Rev. A74 053419 (2006).

12 V. Apaja and O.F. Syljuåsen, Phys Rev A74, 035601 (2006).

13 V.G. Rousseau, Phys. Rev. E77, 056705 (2008); V.G. Rousseau, Phys. Rev. E78, 056707 (2008).

14 G.G. Batrouni and R.T. Scalettar, Phys. Rev. Lett. 84, 1599 (2000).

15 While $\left\langle F^{2}\right\rangle$ vanishes for the even Mott lobes, for the odd lobes $\left\langle F^{2}\right\rangle \rightarrow 2$. This is obvious for $\rho=1$ since there is a single spin- 1 boson on each site. For $\rho=3$, for example, two of the bosons pair into a singlet, again leaving an effective spin- 1 on each site.

${ }^{16}$ In a simulation in the canonical ensemble in the $F_{z}=$ 0 sector, $S_{\mathrm{zz}}(q=0)$ is constrained to vanish at $q=0$. However, its $q \rightarrow 0$ limit gives the $q=0$ values of the unconstrained situation ? $^{\frac{3}{}}$

17 L.E. Sadler et al, Nature 443, 312 (2006).

18 E. Demler and F. Zhou, Phys. Rev. Lett. 88, 163001 (2002); M. Snoek and F. Zhou, Phys. Rev. B69, 094410 (2004). 
19 A. Imambekov, M. Lukin, and E. Demler, Phys. Rev. A68, 063602 (2003) and Phys. Rev. Lett. 93, 120405 (2004). 